\title{
Article
}

\section{Topology Optimization of Multi-Materials Compliant Mechanisms}

\author{
Wenjie Ge * and Xin Kou
}

check for

updates

Citation: Ge, W.; Kou, X. Topology Optimization of Multi-Materials Compliant Mechanisms. Appl. Sci. 2021, 11, 3828. https://doi.org/ 10.3390/app11093828

Academic Editor: Renato Vidoni

Received: 15 March 2021

Accepted: 21 April 2021

Published: 23 April 2021

Publisher's Note: MDPI stays neutral with regard to jurisdictional claims in published maps and institutional affiliations.

Copyright: (c) 2021 by the authors. Licensee MDPI, Basel, Switzerland. This article is an open access article distributed under the terms and conditions of the Creative Commons Attribution (CC BY) license (https:/ / creativecommons.org/licenses/by/ $4.0 /)$.
School of Mechanical Engineering, Northwestern Polytechnical University, Xi'an 710072, China; nwpup@foxmail.com

* Correspondence: kkxx985@mail.nwpu.edu.cn

\begin{abstract}
In this article, a design method of multi-material compliant mechanism is studied. Material distribution with different elastic modulus is used to meet the rigid and flexible requirements of compliant mechanism at the same time. The solid isotropic material with penalization (SIMP) model is used to parameterize the design domain. The expressions for the stiffness matrix and equivalent elastic modulus under multi-material conditions are proposed. The least square error (LSE) between the deformed and target displacement of the control points is defined as the objective function, and the topology optimization design model of multi-material compliant mechanism is established. The oversaturation problem in the volume constraint is solved by pre-setting the priority of each material, and the globally convergent method of moving asymptotes (GCMMA) is used to solve the problem. Widely studied numerical examples are conducted, which demonstrate the effectiveness of the proposed method.
\end{abstract}

Keywords: compliant mechanism; topology optimization; multi-material; volume constraint

\section{Introduction}

A compliant mechanism is a new type of hingeless mechanism that relies on the elastic deformation of the whole or part of the mechanism itself to obtain movement. Due to the characteristics of low noise and vibration, no lubrication, compliant mechanisms are gaining interest in the fields of micro-electro-mechanical systems, medical devices and aerospace $[1,2]$. Topology optimization method is the most widely used and effective tool for designing compliant mechanism [3]. Ananthasuresh et al. [4] introduced the concept of topology optimisation to the design of compliant mechanisms for the first time using the homogenisation method proposed by Bendsøe. Topology optimization is a kind of design method in engineering, which is based on certain external forces and other constraints to seek the best material layout and obtain the structure that meets the specific output requirements. The most important innovation of topology optimization is to find the optimal distribution form in a given design area from a higher dimensional point of view. The variable density method represented by solid isotropic material with penalization (SIMP) has become the mainstream method of topology optimisation due to the great simplification of the design variables and improved computational efficiency and has achieved good results in the design of compliant mechanisms [5-8]. Sigmund et al. used mechanical advantage as an objective function for the design of compliant mechanism [9]. Larsen [10] proposed geometric advantage as a design objective for the variable density method. Frecker et al. [11] proposed the idea of using the ratio of mutual potential energy to strain energy for the design of compliant mechanisms. Xie proposed a design method for compliant mechanisms with mechanical efficiency as the design objective [12]. Bruns started the nonlinear problems in the design of compliant mechanisms [13]. Lin et al. [14] tries to use the maximum output displacement with error constraints. Cho [15] proposed that displacement load can be used to replace force load in optimization, which can improve the convergence rate and obtain the convergence solution easily. Pedersen et al. proposed the design of large displacement compliant mechanisms considering path planning $[16,17]$. 
Bernardoni et al. used compliant structural element to improve the solution speed [18]. Abdalla takes the maximum energy efficiency as the goal of compliant mechanisms [19]. The design of compliant mechanisms with maximum flexibility and minimum stiffness was optimized by Luo et al. [20]. Carbonari et al. [21] proposed a multi-physics field topology optimization method for compliant mechanisms with functional gradients. Rahmatalla proposed an optimization method that includes different virtual spring stiffnesses in the topology optimisation of compliant mechanisms [22]. Hull proposed a sub-region partitioning design method in order to reduce the computational complexity of the compliant mechanism design [23]. Lin et al., proposed a volume self-adjusting algorithm to solve the problem of artificially setting the volume fraction in topology optimization [24]. Lan et al. [25], Zuo et al. [26] used a linear incremental method to reduce the computational complexity by replacing the non-linear problem with a series of linear problems. Jang proposed the method of using standardized elements in compliant mechanisms design [27]. Zhang et al. conducted an in-depth study of multiple input-output and fatigue reliability problems for compliant mechanisms [28-32]. Chen has done a lot of research work on pseudo-rigid body models and flexible hinges for compliant mechanisms [33-36].

The essence of topology optimisation for compliant mechanisms is to find the best layout of materials in the design domain that satisfies the boundaries and constraints, and the properties of the material itself have a significant impact on the design results. However, current research on compliant mechanisms is mainly focused on single materials, which determine that the design results are difficult to meet the requirements of compliant mechanisms applications. In order to maximise the design potential of topology optimisation, it is necessary to develop from the traditional design using single materials to match materials and properties, i.e., topology optimisation of multi-material compliant mechanisms. Thomsen carried out the research on topology optimization of two materials based on the homogenization method for the first time [37]. Yin proposed that the material properties of a multi-material can be described in terms of a single variable through a peak function interpolation model [38]. Sun et al. proposed a microscopic multi-material optimisation design model [39]. The design of a multi-material compliant actuator was investigated by Luo et al. through a level set approach [40]. Topology optimization of thermal expansion compliant mechanisms using two materials were carried out by Sigmund et al. [41,42]. Zhang proposed a solution strategy for multi-objective topology optimization under thermal structural coupling [43,44]. Alonso et al. proposed an element exclusion strategy for multi-material compliant mechanisms to achieve the arrangement of each material in the design domain with different addition and deletion criteria of element materials [45,46]. Zhang et al. used the method of moving asymptotes to solve the problem of variables in multi-material topology optimization [47]. Zuo et al. proposed topology optimization of discrete variable structures under multi-material cost constraints [48]. Tavakoli proposed an Allen-Cahn projection gradient multi-material topology optimization method based on the SIMP model [49]. Tong et al. studied the topology optimization design of multi-material laminates [50]. Guest controlled the length scale by the Heaviside projection method in the topology optimization of multi-material [51]. Jiang presents a systematic design method for a 2-degrees-of-freedom compliant parallel mechanism with decoupling performance, input coupling is reduced by resorting to a flexure-based decoupler [52]. In order to save computational resources, Cravero et al. proposed simple models to simulate the fluid-dynamic behaviour and to predict the instability operating range [53,54]. At present, the design theory of multi-material compliant mechanisms is basically extended from the single material, but the number of design variables is multiplied, and the existing material interpolation models are not efficient. At the same time, due to the introduction of multi-material, the numerical instability of single node hinge and checkerboard is more prominent.

In this paper, based on the generalized SIMP material interpolation model, taking the least square difference between the actual displacement and the target displacement of each output index point as the optimization objective, the mathematical model for topology 
optimization of multi-material compliant mechanisms is established. The polygonal mesh is used in the finite element solution process to avoid single node hinge. The remainder of the paper is organized as following. Section 2 is the main part of this work, the topology optimization model for multi-material compliant mechanisms, as well as the main idea of the proposed method is given. In Section 3, two benchmark numerical examples are provided to illustrate that our method is validity. Section 4 gives some conclusions.

\section{The Topology Optimization Model for Multi-Material Compliant Mechanisms}

\subsection{Generalized SIMP}

In topology optimization, SIMP method drives the intermediate density values of design variables to $0 / 1$ by penalty factor power, which leads to less design variables and a simple calculation. In this paper, the multi-material problem is decomposed into a series of single material subproblems, and the generalized SIMP interpolation model is established to optimize the topology of multi-material compliant mechanisms. For example, the elastic modulus interpolation model of three-phase materials can be expressed as:

$$
E\left(x_{1}, x_{2}\right)=x_{1}^{p} x_{2}^{p} E_{2}+x_{1}^{p}\left(1-x_{2}^{p}\right) E_{1}+\left(1-x_{1}^{p}\right) E_{0}
$$

where $x_{1}$ represents the presence or absence of element material, $x_{2}$ represents which phase material the element material belongs to, $E_{1}$ and $E_{2}$ are the elastic modulus of material 1 and solid material 2 respectively. It is worth noting that the void region is considered a phase material with a very small Young's modulus $E_{0}$. The penalization factor is indicated by $p($ e.g., $p=3)$. Figure 1 shows a random distribution of multi-material in the design domain.

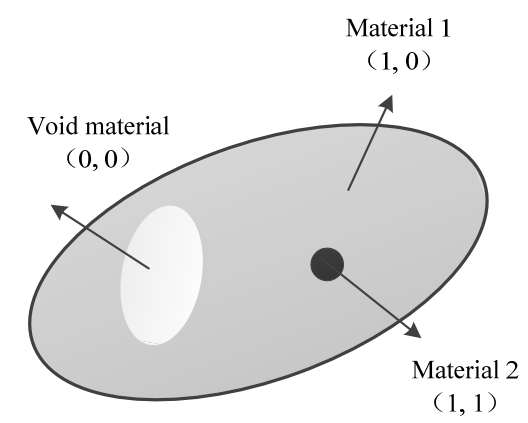

Figure 1. A random distribution of multi-material in the design domain.

The equivalent element stiffness matrix can be obtained from Equation (2), where $M$ is the number of elements, $k$ is the stiffness matrix of each element:

$$
\begin{aligned}
k\left(x_{1}^{e}, x_{2}^{e}, \ldots, x_{M}^{e}\right) & =\sum_{i=1}^{M}\left(x_{i}^{e}\right)^{p} k_{i}^{e} \\
i=1,2, \ldots, M e & =1,2, \ldots, N
\end{aligned}
$$

\subsection{The Element Density Processing Method}

According to SIMP model, any element density value is a value between $0 / 1$. After the SIMP model is extended to the multi-material problem, it is possible that each material exists simultaneously in anyone element, which will lead to the total density of the element being greater than 1 . In order to make the model still valid for multi-material problems, it is necessary to limit the element density. That is, to ensure that the sum of the material densities of any element does not exceed 1 . When the total density of an element exceeds 1 , the material with the lowest density priority in the element is discarded. Assuming that 
the presupposition priority of each material is $P r_{1}, P r_{2}, \ldots, P r_{M}$ in descending order, then the element density value after the processing is:

$$
\begin{gathered}
x_{P r(M-k)}^{e}=\min \left(0,1-\sum_{i=1}^{M-(k+1)} x_{P r_{i}}^{e}\right) \\
\sum_{i=1}^{M-k} x_{P r_{i}}^{e}>1 k=1,2, \ldots, M-1
\end{gathered}
$$

\subsection{Topology Optimization Model and Sensitivity Analysis of Multi-Material Compliant} Mechanisms

Compliant mechanisms often have the requirement of output specific displacement, Figure 2 shows the design model of multi-material compliant mechanisms. The design domain of the mechanism is composed of three materials, which need to produce displacement $u_{\text {out }, i}$ to clamp the target under the action of force $F_{1}$.

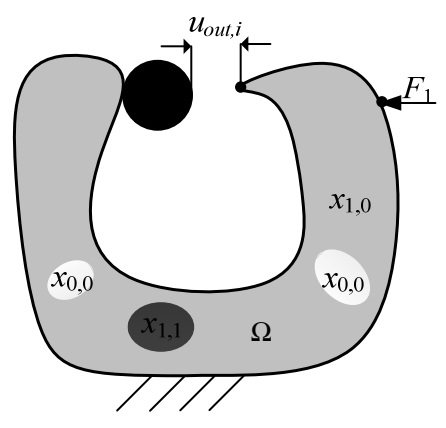

Figure 2. The design model of multi-material compliant mechanisms.

Based on the generalized SIMP material interpolation model, taking the least square difference between the actual displacement and the target displacement of each output index point as the optimization objective, the mathematical model for topology optimization of multi-material compliant mechanisms is shown in Equation (4):

$$
\begin{gathered}
\min : C(x)=\sum_{i=1}^{M} a_{i}\left(u_{\text {out }, i}-u_{\text {out }, i}^{*}\right)^{2}=\sum_{i=1}^{M} a_{i}\left(\sum_{e=1}^{N} x_{e}^{p} \widetilde{u}^{T} \bar{k}^{e} u-u_{\text {out }, i}^{*}\right)^{2} \\
K \bar{U}=F \\
\text { s.t: }\left\{\begin{array}{c}
N \\
V_{x 1}=\sum^{N} x_{1,0} v_{1}<V_{10} \\
V_{x 2}=\sum^{N} x_{1,1} v_{2}<V_{20} \\
0<x_{\min }<x_{1,0}<x_{\max }<1 \\
0<x_{\min }<x_{1,1}<x_{\max }<1
\end{array}\right.
\end{gathered}
$$

where $C(x)$ is the objective function; $U$ and $U$ are the displacement fields of the mechanism under the driving load $F$ and the virtual adjoint load $L$ respectively; $K$ is the global stiffness matrix of the multi-material mechanism; and $V_{x 1}$ and $V_{x 2}$ are the optimized volume of materials $V_{10}$ and $V_{20}$, respectively.

The sensitivity analysis is generally used in SIMP-based optimization problem solving, which refers to the objective function's responsiveness to the design variables. The objective function sensitivity of the multi-material density separately derivation. The sensitivity of the objective function of the first material is as following:

$$
\begin{aligned}
\frac{\partial C}{\partial x_{1,0}} & =F^{T} K^{-1}\left(\frac{\partial F}{\partial x_{1,0}}-\frac{\partial K}{\partial x_{1,0}} U\right) \\
& =-\bar{U}^{T} \frac{\partial K}{\partial x_{1,0}} U
\end{aligned}
$$


The sensitivity of the objective function to the second material can be formulated as:

$$
\begin{aligned}
\frac{\partial C}{\partial x_{1,1}} & =F^{T} K^{-1}\left(\frac{\partial F}{\partial x_{1,1}}-\frac{\partial K}{\partial x_{1,1}} U\right) \\
& =-\bar{U}^{T} \frac{\partial K}{\partial x_{1,1}} U
\end{aligned}
$$

$\partial C / \partial x_{1,0}$ and $\partial C / \partial x_{1,1}$ are expanded as follows:

$$
\begin{aligned}
& \frac{\partial k_{e}}{\partial x_{1,0}}=p x_{1,0}^{p-1}\left(x_{1,1}^{p} E_{2}+\left(1-x_{1,1}^{p}\right) E_{1}-E_{0}\right) \frac{k_{e}}{E\left(x_{1,0}, x_{1,1}\right)} \\
& \frac{\partial k_{e}}{\partial x_{1,1}}=p x_{1,0}^{p} x_{1,1}^{p-1}\left(E_{2}-E_{1}\right) \frac{k_{e}}{E\left(x_{1,0}, x_{1,1}\right)}
\end{aligned}
$$

The process of topology optimization of the multi-material compliant mechanisms is shown in Figure 3. Firstly, the design domain is initialized. secondly, the finite element mesh is divided, the equivalent stiffness matrix and elastic modulus are calculated, then the topology optimization mathematical model of the multi-material material compliant is established and sensitivity analysis is performed, and the design variables are updated by the Globally Convergent Method of Moving Asymptotes (GCMMA ) method; finally, whether convergence is achieved or not is judged, and if convergence is achieved, the optimal result is output otherwise the iteration continues.

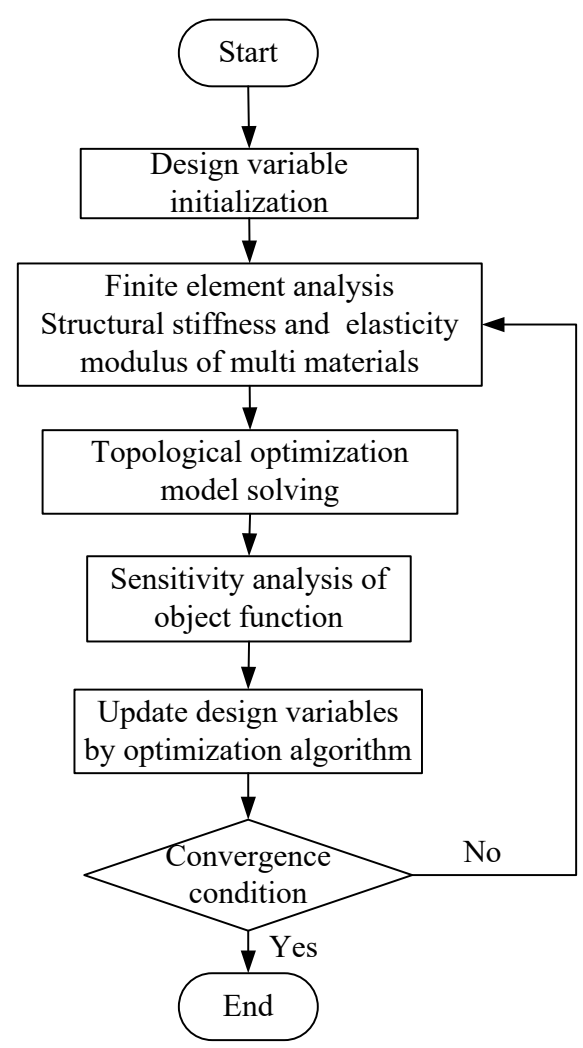

Figure 3. Flowchart of the algorithm.

\section{Results and Discussion}

We present benchmark examples of compliant mechanisms in this section to illustrate the viability and efficiency of the methodology presented in this paper. GCMMA optimization algorithm based on MATLAB platform, is used to obtain the optimal topology of compliant mechanisms. Polygonal discretization (1200 FE elements) can be obtained from the Voronoi diagram, which not to be susceptible to numerical instability such as checkerboard patterns. 


\subsection{Inverter}

The first benchmark example is an inverter with single input and output which is illustrated in Figure 4. The input and output stiffness has an equal value of 0.1. This mechanism is considered to be designed with four material properties. To investigate the effect of multi-material, three different cases for volume fraction are presented. The design parameters are presented in Table 1, that consist of three solid material phases, including polyetherimide, polyurethane and polyvinylidinefluoride (Material 1, Material 2 and Material 3, respectively).

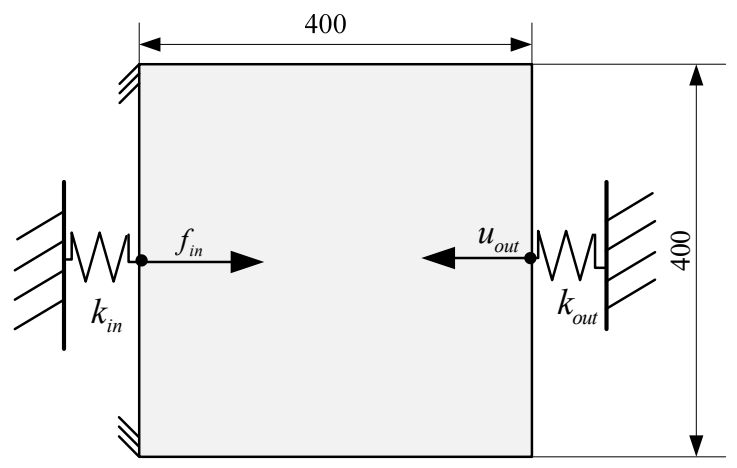

Figure 4. The design domain of a compliant inverter mechanism.

Table 1. Design parameters for the inverter mechanism.

\begin{tabular}{ccccc}
\hline & Material 1 & Material 2 & Material 3 & Material 4 \\
\hline Young's modulus (MPa) & 10 & 5 & 2.5 & $1.0 \times 10^{-9}$ \\
Input force(N) & 3 & 3 & 3 & 3 \\
Poisson ratio & 0.3 & 0.3 & 0.3 & 0.3 \\
Volume fraction Case 1 & 0.3 & 0.01 & 0.01 & 0.68 \\
Volume fraction Case 2 & 0.16 & 0.08 & 0.08 & 0.68 \\
Volume fraction Case 3 & 0.08 & 0.16 & 0.08 & 0.68 \\
Volume fraction Case 4 & 0.08 & 0.08 & 0.16 & 0.68 \\
Color & Red & Black & Blue & White \\
\hline
\end{tabular}

In case 1 , the multi-material method is degenerated into the traditional single material method which only contains real material and empty phase, so as to verify the correctness of the multi-material compliant mechanisms design method proposed in this paper.

To verify the result, after using the equal load conditions, the topology of compliant inverter of traditional SIMP method [55] and degenerate solution of multi-material method was obtained, as showed in Figure $5 \mathrm{a}, \mathrm{b}$ the distribution form of material is basically the same, and differences only in very small localities. In Figure 5c, the output displacement of the optimized result after the degradation of multi-material is $-5.3 \mathrm{~mm}$, which is only $3.7 \%$ error compared with $-5.1 \mathrm{~mm}$ of the traditional single material (see Table 2). The results indicate a slight difference in the maximum displacement of compliant inverter, this shows that the principle and method of compliant mechanism design for multi-material proposed in this paper are correct and effective.

Table 2. Maximum displacement at the output port of inverter.

\begin{tabular}{ccc}
\hline Numerical Simulation & Traditional SIMP Method & Error \\
\hline $5.3 \mathrm{~mm}$ & $5.1 \mathrm{~mm}$ & $3.7 \%$ \\
\hline
\end{tabular}

By observing Figures $6 \mathrm{a}, 7 \mathrm{a}$ and $8 \mathrm{a}$, it can be found that the high modulus material phase is mainly distributed near the fixed end of the mechanism to maintain the shape, the 
medium modulus material is mainly distributed in the deformation guiding and execution area of the compliant mechanism, while the low modulus material is more concentrated in the local deformation area, which can avoid the problem of high local stress. The output displacements in Figures $6 \mathrm{~b}, 7 \mathrm{~b}$ and $8 \mathrm{~b}$ are $-8.4 \mathrm{~mm},-9.4 \mathrm{~mm}$ and $-10.5 \mathrm{~mm}$, respectively, with $13 \%$ increase in deformation capacity when the dominant material in the four phases is changed from high to medium modulus. When the dominant material in the four phases changed from high to low modulus, the deformation ability of the mechanism increased by $25 \%$.

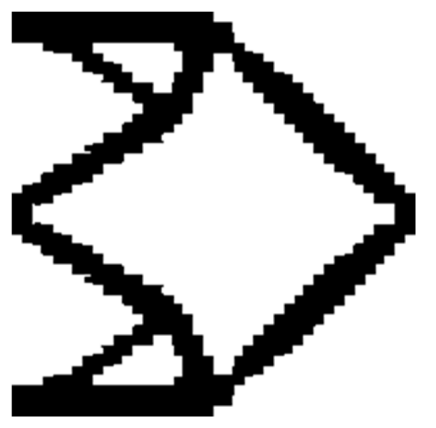

(a)

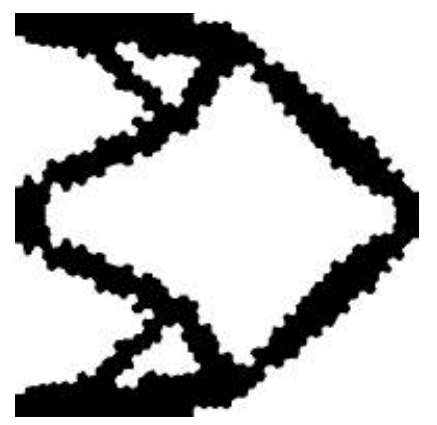

(b)

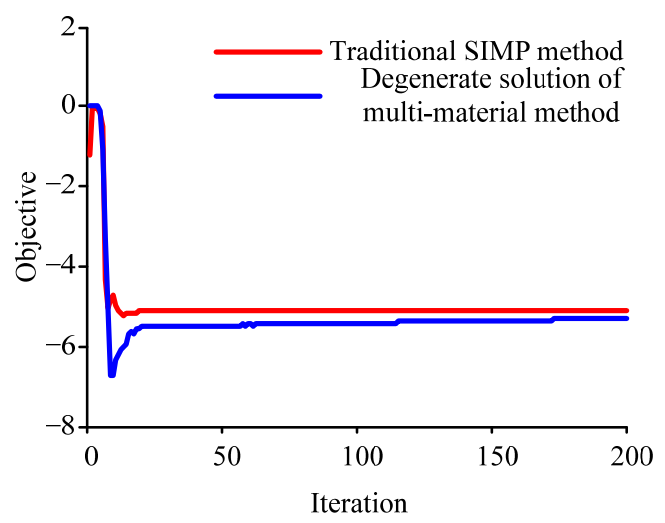

(c)

Figure 5. Optimization results: (a) Traditional SIMP method topologies; (b) degenerate solution of multi-material method (case 1); (c) convergence plot.

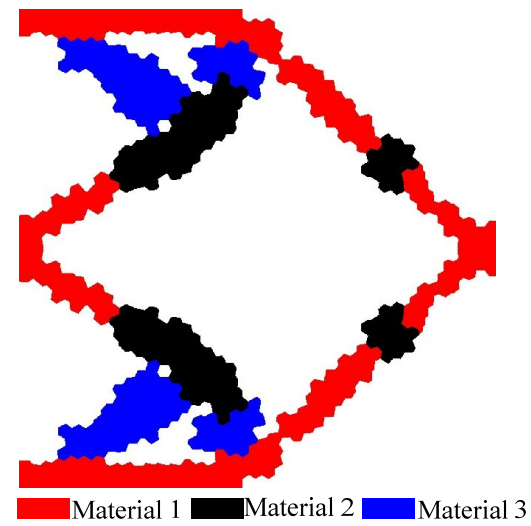

(a)

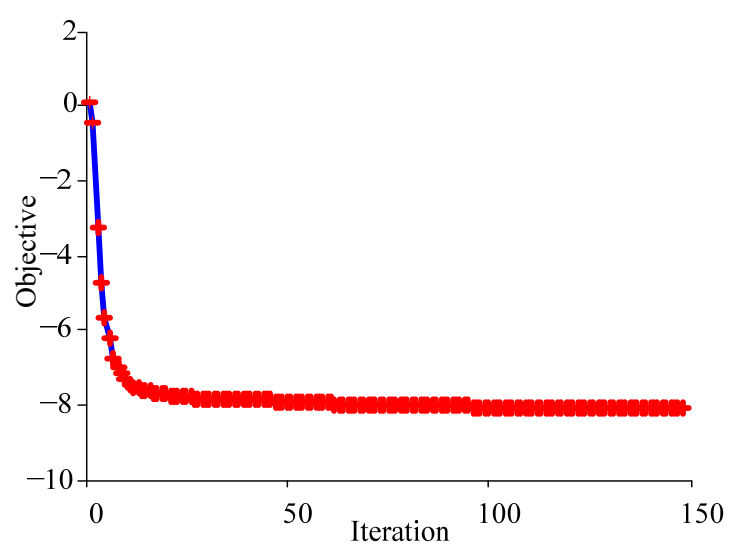

(b)

Figure 6. Optimization results of case 2: (a) Identifed topologies; (b) convergence plot. 


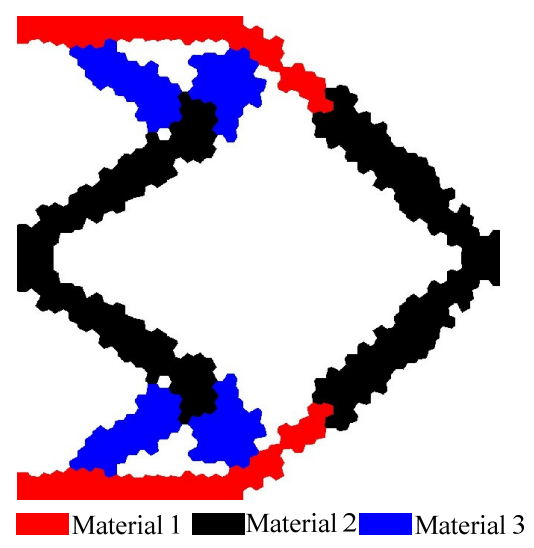

(a)

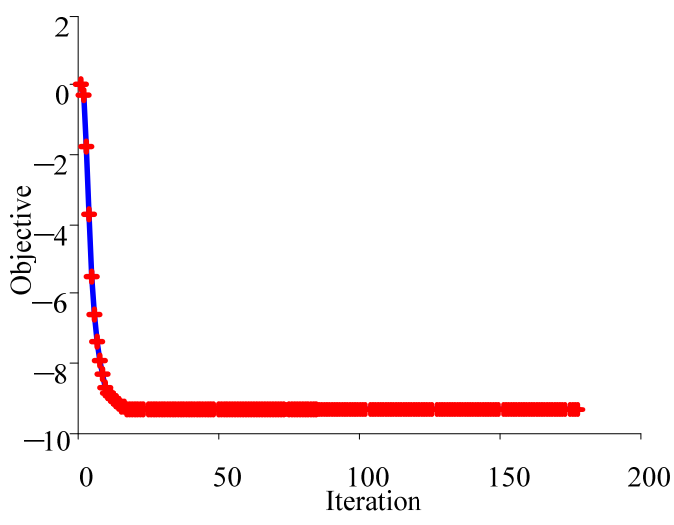

(b)

Figure 7. Optimization results of case 3: (a) Identifed topologies; (b) convergence plot.

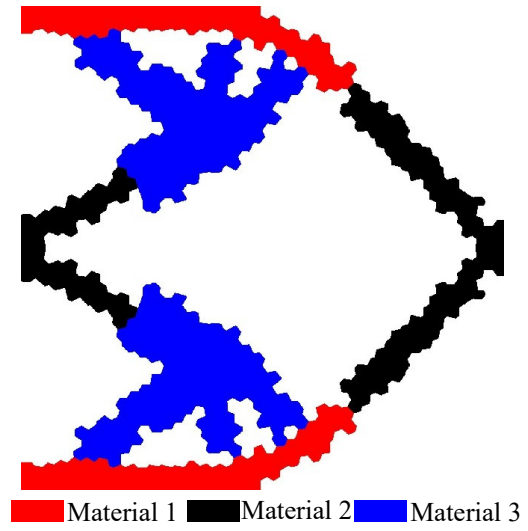

(a)

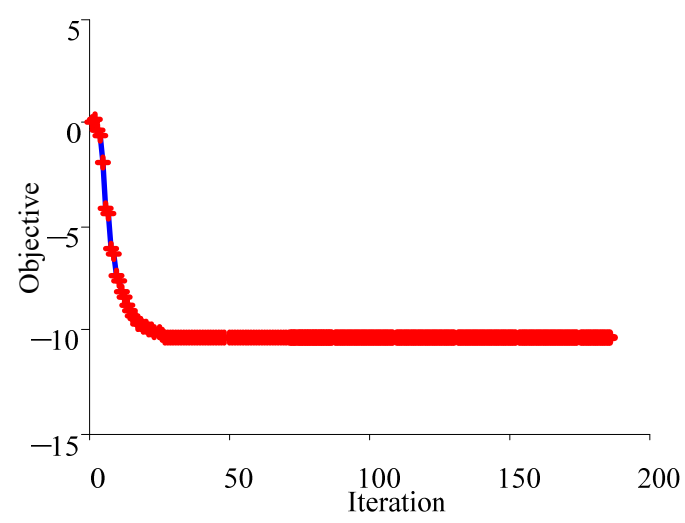

(b)

Figure 8. Optimization results of case 4: (a) Identifed topologies; (b) convergence plot.

\subsection{Compliant Gripper}

The second benchmark problem is the compliant gripper which is illustrated in Figure 9. Again, the design parameters are presented in Table 3.

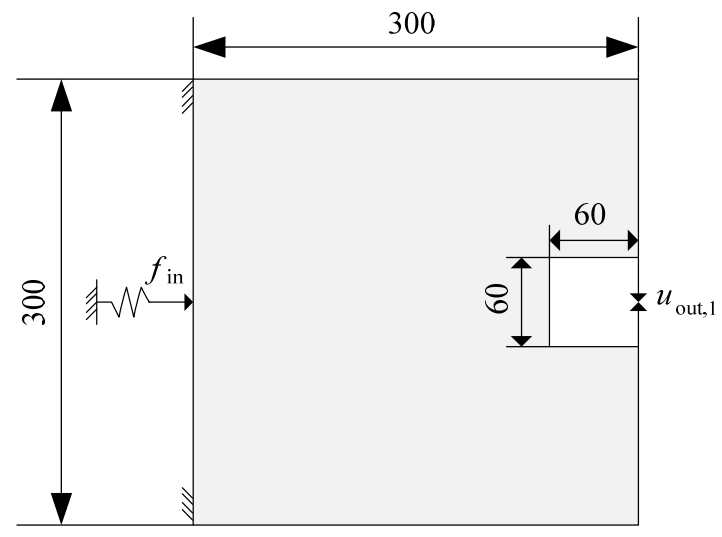

Figure 9. The design domain of a compliant gripper. 
Table 3. Design parameters for the compliant gripper.

\begin{tabular}{ccccc}
\hline & Material 1 & Material 2 & Material 3 & Material 4 \\
\hline Young's modulus (MPa) & 100 & 50 & 25 & $1.0 \times 10^{-9}$ \\
Input force(N) & 3 & 3 & 3 & 3 \\
Poisson ratio & 0.3 & 0.3 & 0.3 & 0.3 \\
Volume fraction Case 1 & 0.3 & 0.01 & 0.01 & 0.68 \\
Volume fraction Case 2 & 0.16 & 0.08 & 0.08 & 0.68 \\
Volume fraction Case 3 & 0.16 & 0.10 & 0.06 & 0.68 \\
Volume fraction Case 4 & 0.06 & 0.16 & 0.10 & 0.68 \\
Color & Red & Black & Blue & White \\
\hline
\end{tabular}

Under equal load conditions, the topology of compliant gripper of traditional SIMP method and degenerate solution of multi-material method was obtained, as showed in Figure 10a,b. The same as before, an inconsiderable difference can be seen between them (shown in Table 4).

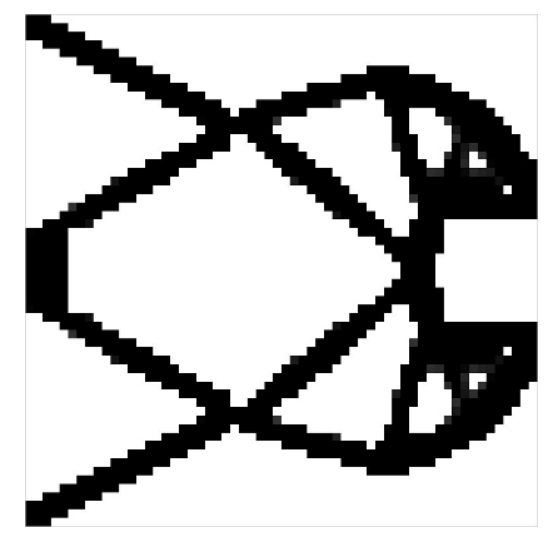

(a)

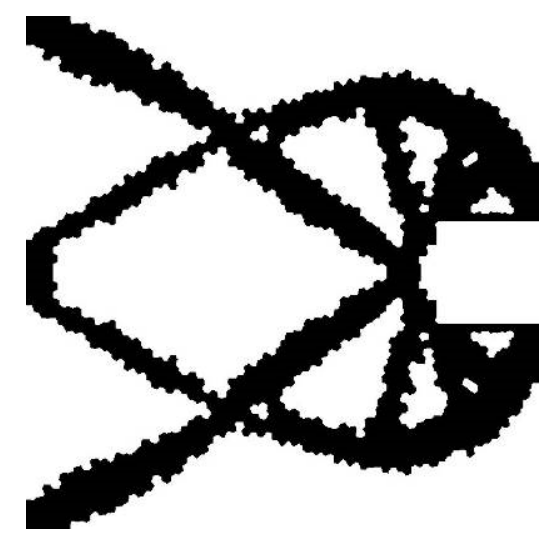

(b)

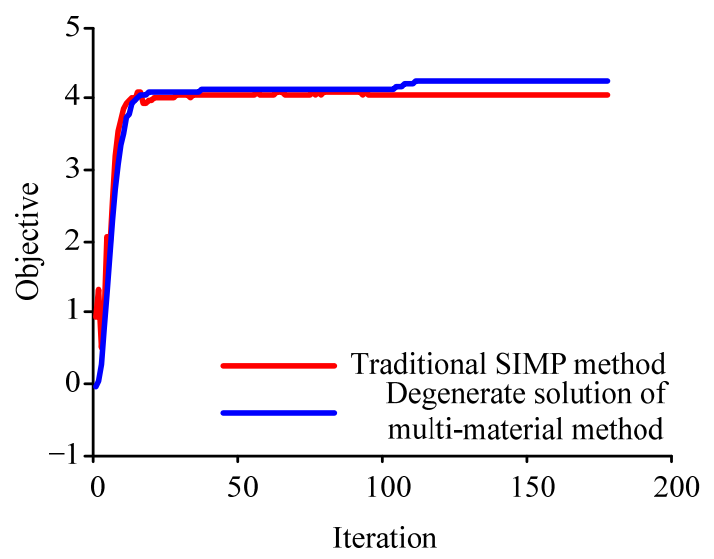

(c)

Figure 10. Optimization results: (a) Traditional SIMP method topologies; (b) degenerate solution of multi-material method (case 1); (c) convergence plot.

Table 4. Maximum displacement at the output port of compliant gripper.

\begin{tabular}{ccc}
\hline Numerical Simulation & Traditional SIMP Method & Error \\
\hline $4.23 \mathrm{um}$ & $4.1 \mathrm{um}$ & $3.2 \%$ \\
\hline
\end{tabular}


The results of topology optimization under the three design schemes of compliant gripper are shown in Figures 11-13, respectively.

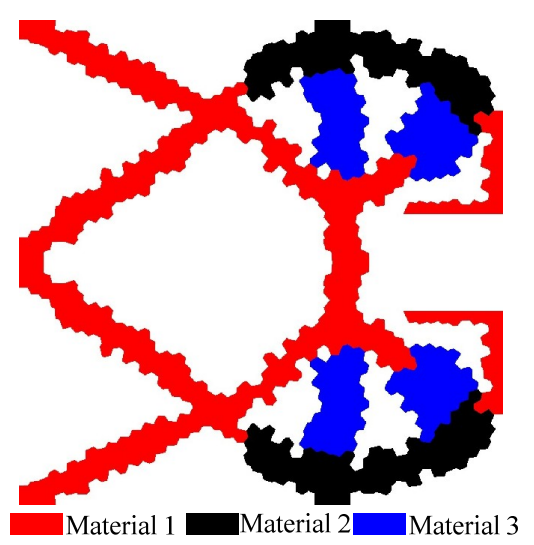

(a)

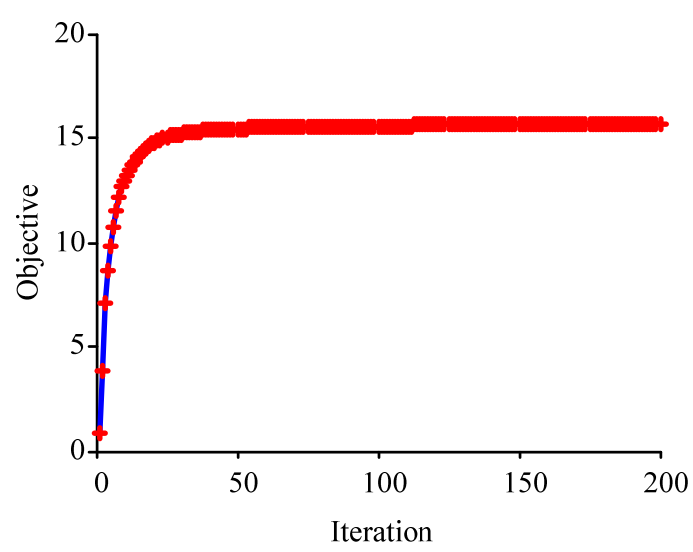

(b)

Figure 11. Optimization results of gripper case 2: (a) Identifed topologies; (b) convergence plot.

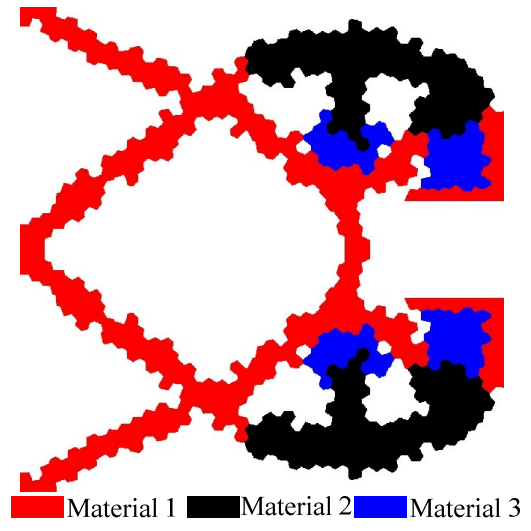

(a)

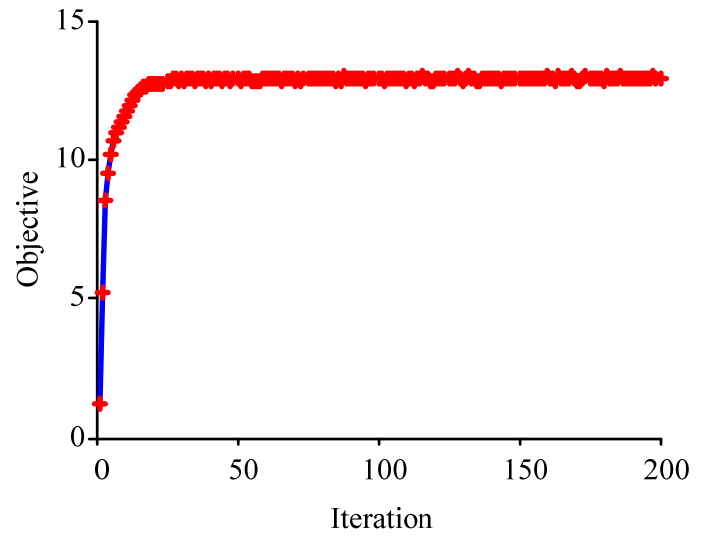

(b)

Figure 12. Optimization results of gripper case 3: (a) Identifed topologies; (b) convergence plot.

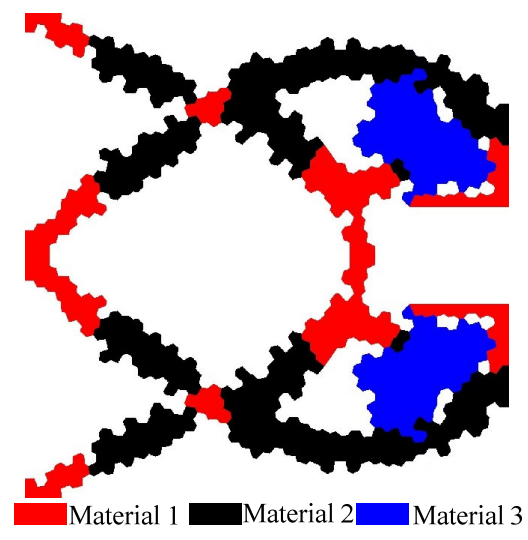

(a)

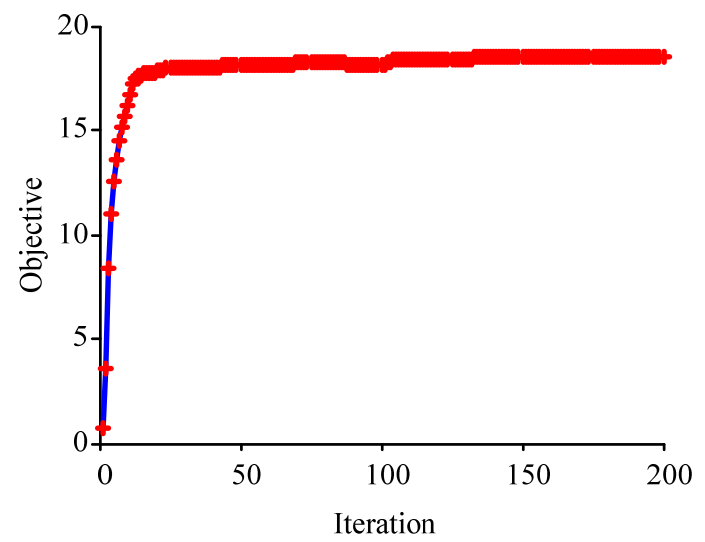

(b)

Figure 13. Optimization results of gripper case 4: (a) Identifed topologies; (b) convergence plot.

It can be seen from the results that each material distribution of the three schemes is relatively independent, and the boundary is clear and easy to identify. Compared with 
Figures 11a and 12a, keeping the volume ratio of high modulus and empty phase materials unchanged, increasing the medium modulus and reducing the ratio of low modulus materials, the topological distribution of each material in the compliant clamp is basically the same. However, the output displacement is reduced as showing in Figures 11b and 12b. The displacement of case 2 is $15.7 \mathrm{um}$, and that of case 3 is $12.9 \mathrm{um}$. The deformation capacity of the mechanism decreases by $17.7 \%$, which reflects the essential influence of the elastic modulus of the material on the deformation capacity of the compliant mechanism. By observing Figures 12a and 13a, when the dominant material changes from high/medium modulus of case 3 to medium/low modulus of case 4 , the topological distribution of the four-phase material of the compliant clamp changes obviously, and the distribution of the medium modulus material is more uniform, which is conducive to the overall deformation of the compliant mechanism. In Figure 13b, the output displacement of case 4 is $18.4 \mathrm{um}$, and the deformation capacity of the mechanism is increased by $42.3 \%$.

\section{Conclusions}

In this paper, the generalized SIMP interpolation model was utilized to design complicated multi-material compliant mechanisms. The oversaturation problem in the volume constraint is solved by pre-setting the priority of each material. LSE between the deformed and target displacement of the control points is defined as the objective function, and the topology optimization design model of multi-material compliant mechanism is established. Two benchmark examples are considered in this paper. The designs are produced with different material and volume fractions. Compared with the results of conventional single material topology optimization, the flexible deformation ability of both examples is improved, and a better mechanism configuration is obtained. The agreement between the results implies the capability of the method to design multi-material compliant mechanisms. The method is quite simple in application and can handle any kind of material with clear chunks between each, although construction of these multi-material mechanisms is not easy, the final distribution of material can be inspiring designers.

Author Contributions: Conceptualization: W.G. and X.K.; methodology: X.K.; software: X.K.; validation: X.K. and W.G.; formal analysis: X.K. and W.G.; investigation: X.K. and W.G.; resources: W.G.; data curation: X.K.; writing—original draft preparation: X.K.; writing—review and editing: X.K. and W.G.; supervision: W.G; project administration: W.G.; funding acquisition: W.G. All authors have read and agreed to the published version of the manuscript.

Funding: This research was funded by the National Natural Science Foundation of China, grant number 51375383.

Institutional Review Board Statement: Not applicable.

Informed Consent Statement: Not applicable.

Data Availability Statement: Not applicable.

Conflicts of Interest: The authors declare no conflict of interest.

\section{References}

1. Howell, L.L. Compliant Mechanisms, 1st ed.; John Wiley \& Sons Limited: Manhattan, NY, USA, $2001 ;$ pp. 18-51.

2. Burns, R.H.; Crossley, F.R. Kinetostatic synthesis of flexible link mechanisms. Mech. Eng. 1968, 90, 67.

3. Sigmund, O. On the Design of Compliant Mechanisms Using Topology Optimization. Mech. Struct. Mach. 1997, $25,493-524$. [CrossRef]

4. Ananthasuresh, G.K.; Kota, S.; Kikuchi, N. Strategies for systematic synthesis of compliant MEMS. Dyn. Syst. Control 1994, 55, 677-686.

5. Mlejnek, H.P. Some aspects of the genesis of structures. Struct. Optim. 1992, 5, 64-69. [CrossRef]

6. Yang, R.J. Topology optimization analysis with multiple constraints. Am. Soc. Mech. Eng. 1995, 82, $393-398$.

7. Jian, W.; Cheng, G.D. Optimal Topology Design of Thin Plate With Stress Constraints. Acta Mech. Solida Sin. 1997, 18, 37-42.

8. Yuan, Z.; Wu, C.C.; Zhuang, S.B. Topology Optimization of Continuum Structure Using Hybrid Elements and Ariticial Material Model. J. Univ. Sci. Technol. China 2001, 31, 694-699. 
9. Sigmund, O. Design of multiphysics actuators using topology optimization-part ii: Two-material structures. Comput. Methods Appl. Mech. Eng. 2001, 190, 6605-6627. [CrossRef]

10. Larsen, U.D.; Signund, O.; Bouwsta, S. Design and fabrication of compliant micromechanisms and structures with negative Poisson's ratio. J. Microelectromech. Syst. 1997, 6, 99-106. [CrossRef]

11. Frecker, M.I.; Ananthasuresh, G.K.; Nishiwaki, S.; Kikuchi, N.; Kota, S. Topological synthesis of compliant mechanisms using multi-criteria optimization. J. Mech. Des. 1997, 119, 238-245. [CrossRef]

12. Xie, X.H.; Liao, D.X. On the Design of Compliant Mechanisms Using Homogenization Method. China Mech. Eng. 2003, 14, 953-955.

13. Bruns, T.E.; Tortorelli, D.A. Topology optimization of non-linear elastic structures and compliant mechanisms. Comput. Methods Appl. Mech. Eng. 2001, 190, 3443-3459. [CrossRef]

14. Lin, C.F.; Shih, C.J. Topological optimum design of a compliant mechanism for planar optical modulator. Tamkang J. Sci. Eng. 2002, 5, 151-158. [CrossRef]

15. Cho, S.; Jung, H.S. Design sensitivity analysis and topology optimization of displacement loaded non-linear structures. Comput Methods Appl. Mech. Eng. 2003, 2539-2553. [CrossRef]

16. Pedersen, C.B.W. Crashworthiness design of transient frame structures using topology optimization. Comput. Methods Appl. Mech. Eng. 2004, 193, 653-678. [CrossRef]

17. Pedersen, C.B.W.; Buhl, T.; Sigmund, O. Topology synthesis of large displacement compliant mechanisms. Int. J. Numer. Methods Eng. 2001, 50, 2683-2705. [CrossRef]

18. Bernardoni, P.; Riwan, A.; Tsitsiris, H.; Millet, O.; Bidaud, P. From the mechanical analysis of a polyarticulated microgripper to the design of a compliant microgripper. Proc. SPIE 2004, 5383. [CrossRef]

19. Abdalla, M.; Frecker, M.; Gürdal, Z.; Johnson, T.; Lindner, D.K. Design of a piezoelectric actuator and compliant mechanism combination for maximum energy efficiency. Smart Mater. Struct. 2005, 14, 1421. [CrossRef]

20. Luo, Z.; Chen, L.; Yang, J.; Zhang, Y.; Abdel-Malek, K. Compliant mechanism design using multi-objective topology optimization scheme of continuum structures. Struct. Multidiscip. Optim. 2005, 30, 142-154. [CrossRef]

21. Carbonari, R.C.; Nader, G.; Nishiwaki, S.; Silva, E.C.N. Experimental and numerical characterization of multi-actuated piezoelectric device designs using topology optimization. Int. Soc. Opt. Eng. 2005, 5764, 472-481. [CrossRef]

22. Rahmatalla, S.; Swan, C.C. Sparse monolithic compliant mechanisms using continuum structural topology optimization. Int. J. Numer. Methods Eng. 2005, 62, 1579-1605. [CrossRef]

23. Hull, P.V.; Canfield, S. Optimal synthesis of compliant mechanisms using subdivision and commercial FEA. J. Mech. Des. 2006, 128, 337-348. [CrossRef]

24. Lin, C.Y.; Hsu, F.M. An adaptive volume constraint algorithm for topology optimization with a displacement-limit. Adv. Eng. Softw. 2008, 39, 973-994. [CrossRef]

25. Lan, C.C. Analysis of large-displacement compliant mechanisms using an incremental linearization approach. Mech. Mach. Theory 2008, 43, 641-658. [CrossRef]

26. Zuo, K.T.; Chen, L.P.; Wang, S.T.; Zhang, Y.; Zhong, Y. Research on Design of Micro-Compliant Mechanisms with Topology Optimization Method. China Mech. Eng. 2004, 15, 1886-1890.

27. Jang, G.W.; Kim, M.J.; Kim, Y.Y. Design optimization of compliant mechanisms consisting of standardized elements. J. Mech. Des. 2009, 131, 121006. [CrossRef]

28. Zhang, X.M. Topology optimozation of compliant mechanisms. Chin. J. Mech. Eng. 2003, 39, 47-51. [CrossRef]

29. Zhang, X.M.; Chen, Y.J. Topology Optimization Of Multiple Inputs And Outputs Compliant Mechanism with Coupling Terms Control. Chin. J. Mech. Eng. 2006, 42, 162-165. [CrossRef]

30. Li, Z.K.; Zhang, X.M.; Chen, J.Y. Multiobjective Topology Optimization Of Compliant Mechanisms with Geometrical Nonlinearity. J. Mech. Strength 2011, 33, 548-553. [CrossRef]

31. Zhang, X.M.; Wang, Q.L. Research Progress of Fatigue Reliability and Damage Identification of Compliant Mechanisms. J. South China Univ. Technol. 2012, 40, 190-197. [CrossRef]

32. Zhu, B.; Zhang, X.; Wang, N. Topology optimization of hinge-free compliant mechanisms with multiple outputs using level set method. Struct. Multidiplinary Optim. 2013, 47, 659-672. [CrossRef]

33. Chen, G.; Liu, X.; Du, Y. Elliptical-arc-fillet flexure hinges: Toward a generalized model for commonly used flexure hinges. J. Mech. Des. 2011, 133, 081002. [CrossRef]

34. Chen, G.; Wilcox, D.L.; Howell, L.L. Fully compliant double tensural tristable micromechanisms (dttm). J. Micromech. Microeng. 2009, 19, 025011. [CrossRef]

35. Li, G.; Chen, G. A function for characterizing complete kinetostatic behaviors of compliant bistable mechanisms. Mech. Sci. 2014, 5, 67-78. [CrossRef]

36. Chen, G.; Ma, F.; Bai, R.; Magleby, S.P.; Howell, L.L. A Framework for Energy-Based Kinetostatic Modeling of Compliant Mechanisms. In Proceedings of the ASME 2017 International Design Engineering Technical Conferences and Computers and Information in Engineering Conference, Cleveland, OH, USA, 6-9 August 2017.

37. Thomsen, J. Topology optimization of structures composed of one or two materials. Struct. Optim. 1992, 5, 108-115. [CrossRef]

38. Yin, L.; Ananthasuresh, G.K. Topology optimization of compliant mechanisms with multiple materials using a peak function material interpolation scheme. Struct. Multidiscip. Optim. 2001, 23, 49-62. [CrossRef] 
39. Sun, S.P.; Zhang, W.H. Multiple objective topology optimal design of multiphase microstructures. Chin. J. Theor. Appl. Mech. 2006, 38, 633. [CrossRef]

40. Luo, Z.; Tong, L.; Luo, J.; Wei, P.; Wang, M.Y. Design of piezoelectric actuators using a multiphase level set method of piecewise constants. J. Comput. Phys. 2009, 228, 2643-2659. [CrossRef]

41. Sigmund, O.; Torquato, S. Design of materials with extreme thermal expansion using a three-phase topology optimization method. J. Mech. Phys. Solids 1997, 45, 1037-1067. [CrossRef]

42. Gibiansky, L.V.; Sigmund, O. Multiphase composites with extremal bulk modulus. J. Mech. Phys. Solids 2000, 48, 461-498. [CrossRef]

43. Zhang, X.M.; Hu, K.; Wang, N.; Zhang, B. Multi-objective topology optimization of multiple materials compliant mechanisms based on parallel strategy. J. Mech. Eng. 2016, 52, 1-8. [CrossRef]

44. Zhang, X.M.; Hu, K.; Wang, N.F. Design of Thermal-Mechanical Coupling Multiple-Material Compliant Mechanisms Based on Parallel Strategy. J. South China Univ. Technol. 2016, 44, 22-29. [CrossRef]

45. Alonso, C.; Ansola, R.; Querin, O.M. Topology synthesis of multi-material compliant mechanisms with a sequential element rejection and admission method. Finite Elem. Anal. Des. 2014, 85, 11-19. [CrossRef]

46. Alonso, C.; Querin, O.M.; Ansola, R. Topology synthesis of multi-input-multi-output compliant mechanisms. Adv. Eng. Softw. 2014, 76, 125-132. [CrossRef]

47. Zhang, W.; Song, J.; Zhou, J. Topology optimization with multiple materials via moving morphable component (MMC) method. Int. J. Numer. Methods Eng. 2018, 113, 1653-1675. [CrossRef]

48. Zuo, W.; Saitou, K. Multi-material topology optimization using ordered simp interpolation. Struct. Multidiscip. Optim. 2016, 55, 477-491. [CrossRef]

49. Tavakoli, R. Multimaterial topology optimization by volume constrained Allen-Cahn system and regularized projected steepest descent method. Comput. Methods Appl. Mech. Eng. 2014, 276, 534-565. [CrossRef]

50. Tong, X.X.; Ge, W.J.; Zhang, Y.H. Topology design and analysis of compliant mechanisms with composite laminated plates. J. Mech. Sci. Technol. 2019, 33, 613-620. [CrossRef]

51. Guest, J.K. Topology optimization with multiple phase projection. Comput. Methods Appl. Mech. Eng. 2009, 199, 123-135. [CrossRef]

52. Jiang, Y.; Li, T.; Wang, L.; Chen, F. Systematic Design Method and Experimental Validation of a 2-DOF Compliant Parallel Mechanism with Excellent Input and Output Decoupling Performances. Appl. Sci. 2017, 7, 591. [CrossRef]

53. Cavero, C.; Marsano, D. Criteria for the Stability Limit Prediction of High Speed Centrifugal Compressors With Vaneless Diffuser: Part I-Flow Structure Analysis. In Proceedings of the ASME Turbo Expo 2020: Turbomachinery Technical Conference and Exposition. Volume 2E: Turbomachinery, Virtual, Online, 21-25 September 2020. [CrossRef]

54. Cravero, C.; Marsano, D. Criteria for the Stability Limit Prediction of High Speed Centrifugal Compressors With Vaneless Diffuser: Part II-The Development of Prediction Criteria. In Proceedings of the ASME Turbo Expo 2020: Turbomachinery Technical Conference and Exposition. Volume 2E: Turbomachinery, Virtual, Online, 21-25 September 2020. [CrossRef]

55. Kou, X. Topology Optimization of Multiple Outputs Compliant Mechanism with Geometrically Nonlinearity. Master's Thesis, Northwestern Polytechnical University, Xi'an, China, 2010. 